\title{
An in vitro study of vascular endothelial toxicity of CdTe quantum dots
}

\author{
Ming Yan ${ }^{1}$, Yun Zhang ${ }^{1}$, Kedi Xu ${ }^{1}$, Tao Fu ${ }^{2}$, Haiyan Qin ${ }^{2}$, Xiaoxiang Zheng ${ }^{1,3}$
}

\section{Abstract}

Quantum dots (QDs), as novel bioimaging and drug delivery agents, are generally introduced into vascular system by injection, and thus directly exposed to vascular endothelial cells (ECs). However, the adverse effects of QDs on ECs are poorly understood. In this study, employing human umbilical vein ECs (HUVECs), we investigated the potential vascular endothelial toxicity of mercaptosuccinic acid (MSA)-capped CdTe QDs in vitro. In the experiment, water-soluble and $\mathrm{pH}$ stable CdTe QDs were synthesized; and the cell viability assays showed that CdTe QDs (0.1-100 $\mu \mathrm{g} / \mathrm{mL})$ dose-dependently decreased the cell viability of HUVECs, indicating CdTe QDs induced significant endothelial toxicity. The flowcytometric and immunofluorescence results revealed that $10 \mu \mathrm{g} / \mathrm{mL}$ CdTe QDs elicited significant oxidative stress, mitochondrial network fragmentation as well as disruption of mitochondrial membrane potential ( $\left.\Delta \psi_{m}\right)$; whereas ROS scavenger could protect HUVECs from QDs-induced mitochondrial dysfunction. Moreover, upon $24 \mathrm{~h}$ exposure to $10 \mu \mathrm{g} / \mathrm{mL}$ CdTe QDs, the apoptotic HUVECs dramatically increased by 402.01\%, accompanied with alternative expression of apoptosis proteins, which were

\footnotetext{
${ }^{1}$ Department of Biomedical Engineering, Key Laboratory of Biomedical Engineering of Ministry of Education, Zhejiang University, Zheda Road 38, Hangzhou 310027, China

${ }^{2}$ Joint Research Center of Photonics of the Royal Institute of Technology (Sweden) and Zhejiang University, Hangzhou 310058, China

${ }^{3}$ Corresponding author: Dr. Xiaoxiang Zheng, Department of Biomedical Engineering, College of biomedical engineering \& instrument science, Zhejiang University, Hangzhou, China, Fax: 86- 571-87951091, E-mail address: zxx@mail.bme.zju.edu.cn
} 
upregulation of Bax, downregulation of $\mathrm{Bcl}-2$, release of mitochondrial cytochrome c and cleavage of caspase-9/caspase-3. These results suggested that CdTe QDs could not only impair mitochondria but also exert endothelial toxicity through activation of mitochondrial death pathway and induction of endothelial apoptosis. Our results provide strong evidences of the direct toxic effects of QDs on human vascular ECs, and reveal that exposure to QDs is a significant risk for the development of cardiovascular diseases. These results also provide helpful guidance on the future safe use and manipulation of QDs to make them more suitable tools in nanomedicine.

Key words: quantum dots; vascular endothelial cells; apoptosis; mitochondrial dysfunction; reactive oxygen species.

\section{Introduction}

Semiconductor nanocrystals, which are referred to as quantum dots (QDs), have gain much attention since it was first introduced by Louis Brus and Alexi Ekimov in 1980s (Ekimov et al. 1985; Rossetti et al. 1983). These "artificial atoms" exhibit unique electronic and optical properties such as tunable band gaps, high quantum yields, broad absorption spectra, narrow emission spectra and high resistance to photobleaching. During the past decade, QDs have been developed for a broad range of applications. In the optoelectronic fields, great success has been achieved of using QDs in solar energy conversion, light emitting diodes (LEDs), lasers, and display devices (Coe et al. 2002; Huffaker et al. 1998; Huynh et al. 2002; Likharev 1999). 
Meanwhile, in the biomedicine fields, QDs are also successfully utilized as biomedical imaging or traceable drug delivery agents to label biological molecules, cells and even tissues (Resch-Genger et al. 2008), or to follow drug molecules in live organisms (Bagalkot et al. 2007; Manabe et al. 2006). With the spring up of the nascent QD industry, human being is inevitably exposed to these engineered nanomaterials and the studies on the potential toxicity of manufactured QDs become urgent and crucial. Recently, a number of in vitro studies have evaluated the toxicity of QDs in different types of cells. For example, Tang et al. tested the neurotoxicity of CdSe QDs in a hippocampal neuronal culture model and a dose-dependent cell death was observed with 24 h continuously exposure to QDs (Tang et al. 2008). Core QDs and QDs with different coatings were also reported to be toxic to primary rat hepatocytes (Shiohara et al. 2004). In addition, the cadmium based QDs were demonstrated to induce dramatic cell loss in pancreatic carcinoma cells (PANC-1), rat pheochromocytoma cells (PC12) and murine microglial cells (N9) (Chang et al. 2009; Lovric et al. 2005). Although these toxicological studies have shown the toxicity results of cell death for some primary or immortalized cell lines (Derfus et al. 2004; Su et al. 2009; Tang et al. 2008), the understanding of QDs cytotoxicity remains limited.

Lately, QDs were reported to label dermal, adipose, tumor and retinal vasculature (Cai et al. 2006; Jayagopal et al. 2007; Larson et al. 2003). These angiographic techniques provide noninvasive optical access to the vasculature and continuous monitoring of vascular functions. Moreover, Manabe et al. injected QD-conjugated captopril (anti-hypertensive drug) into stroke-prone spontaneously hypertensive rats 
(SHRSP) and analyzed their kinetics and dynamics (Manabe et al. 2006). QDs therefore appear to be a powerful tool for elucidating the pharmacokinetics and pharmacodynamics of drug candidates. However, these novel techniques raised concerns about QDs toxicity on the toxin-susceptible vascular system, especially on the endothelium. Because QDs are need to be directly injected into vascular for these applications, and thus may interact with and impair vascular endothelial cells (ECs), which line the luminal surface of all blood vessels.

The vascular ECs represent a dynamic interface between the circulatory system and the nonvascular tissues, and therefore protect nonvascular tissues such as brain from harmful substances (Lum and Malik 1994). Besides their barrier function, they also play an important role in maintaining vascular homeostasis by releasing various factors to influence vascular tone, thrombogenesis, inflammation, and vessel growth and remodeling (Cines et al. 1998). Endothelial dysfunction and/or apoptosis, whether caused by physical injury or toxicants-induced cellular damage, are considered as early pathological features and independent predictors of poor prognosis in most forms of cardiovascular diseases (CVDs) (Choy et al. 2001; Halcox et al. 2002). Hence, the potential injuries of vascular ECs by QDs are expected to be cardiovascular risks for human. Furthermore, because mitochondrion is a key organelle in conversion of energy, regulation of cellular signaling as well as amplification of programmed cell death in eukaryotic cells including ECs, mitochondrial impairment is believed to induce endothelial dysfunction and promote a series of processes that initiate and exacerbate atherosclerosis (Davidson and Duchen 
2007; Duchen 1999). Previous studies have shown that mitochondria participate in the QDs-induced apoptotic insults to several cell types (Choi et al. 2007; Lovric et al. 2005b). However, the questions of whether QDs can directly affect mitochondrial function, activate mitochondrial death pathways or even trigger apoptosis in ECs remain unanswered.

In this study, we synthesized mercaptosuccinic acid (MSA)-capped CdTe QDs directly via in aqueous condition, and investigated the direct effects of these CdTe QDs on endothelial injury, especially focusing on mitochondrial function and apoptosis. MSA-capped CdTe QDs were selected for this study because they have narrow band gaps and wide Bohr exciton radius, which equip them smaller sizes than most of CdSe QDs for red or near infrared (NIR) emission (Yu et al. 2003); and this enables CdTe QDs with different emission spectra tuned in a broader range can be effectively resolved over the same size range. Our QDs also have favorable pH-stability which supported resistance to the degradation under complicated biological environments. HUVECs were selected in our experiments as an in vitro model to assess the vascular toxicity of CdTe QDs. We found that CdTe QDs induced an increase in intracellular reactive oxygen species (ROS) generation, significant mitochondrial dysfunction, activated the mitochondrial death pathways and induced apoptotic cell death in human vascular ECs. Our results suggested that QDs in circulation could be a strong cardiovascular risk, and provide helpful guidance on the future safe use of QDs. Understanding the mechanisms underlying QD-toxicity will also help researchers improve the eventual design and manipulation of QDs to make 
them more suitable tools in nanomedicine.

\section{Materials and methods}

\subsection{Chemicals and aterials}

Cadmium chloride $\left(\mathrm{CdCl}_{2}, 99.0 \%\right)$, Tellurium powder (Te, 99.5\%) were from Alfa Aesar (London, UK). Sodium borohydride (96\%), hydrochloric acid ( $\mathrm{HCl}$ ) and sodium hydroxide $(\mathrm{NaOH})$ were from Sinopharm Chemical Reagent Co., Ltd (Shanghai, China). All chemicals were used without further purification. Solutions were prepared using Milli-Q water (Millipore) as the solvent. Mercaptosuccinic acid (MSA) was from Sigma-Aldrich Crop. (St. Louis, USA).

Medium 200 (M200), low serum growth supplement (LSGS), fetal bovine serum (FBS), calcein-AM, Annexin V apoptosis detection kit, MitoTracker Red FM and 5,5',6,6'-tetrachloro-1,1',3,3' tetraethylbenzimidazolylcarbocyanine iodide (JC-1) were from Invitrogen Corp. (Carlsbad, USA). The antibodies including anti-human Bcl-2, anti-human Bax, anti-human cytochrome $c$, anti-human caspase-9 and anti-human caspase-3 were from Santa Cruz Biotechnology (Santa Cruz, USA). 2',7'-dichlorfluorescein-diacetate (DCFH-DA) was from Beyotime Institute of Biotechnology (Jiangsu, China). The 3,3,5,5-Tetramethylbenzidine (TMB) Liquid Substrate kit was from Amresco, Inc. (Solon, USA). The secondary antibody and other biological reagents were from Sigma-Aldrich Crop (St. Louis, USA)

\subsection{Preparation and characterization of quantum dots}

The CdTe QDs were synthesized based on the reaction of $\mathrm{CdCl}_{2}$ and $\mathrm{NaHTe}$ solution with MSA as surfactant (Fu et al. 2010). First, $\mathrm{Cd}^{2+}$-MSA precursor solution 
were prepared by dissolving $\mathrm{CdCl}_{2}(0.184 \mathrm{~g})$ and MSA $(0.183 \mathrm{~g})$ in $60 \mathrm{~mL}$ deionized ultra-pure water. The $\mathrm{pH}$ of the solution was adjusted to 6.5 using $1 \mathrm{M} \mathrm{NaOH}$ solution. This mixture was degassed and kept stirring at room temperature. Then, NaHTe solution (4 mL, $0.05 \mathrm{mM}$ ), which was prepared by dissolving Te powder in $\mathrm{NaBH}_{4}$ solution, was injected into the solution. The reaction between cadmium ions and NaHTe took place immediately after the injection of NaHTe and the color of the solution turned red instantly. The molar ratio of $\mathrm{Cd}^{2+}$ : NaHTe: MSA was 1:0.2:1.2. Next, the solution was the CdTe precursor crude solution was refluxed under open-air condition at the temperature $100{ }^{\circ} \mathrm{C}$. The as-prepared QDs were precipitated with an equivalent amount of 2-propanol, and then resuspended in water and precipitated with 2-propanol three more times. The pellet of purified QDs was dried overnight at room temperature in vacuum, and the final product in the powder form could be redissolved in water. The CdTe QDs of powder form could be redissolved in water. Stock solutions of QDs for our following biological experiments were prepared by dissolving CdTe QDs in sterilized phosphate buffered saline (PBS, $\mathrm{pH}=7.4$ ) and filter sterilized through $0.22 \mu \mathrm{m}$ filter.

The high resolution transmission electron microscopy (HRTEM) image was taken by a JEM 2010 microscope (JEOL, Japan) with an acceleration voltage of $200 \mathrm{kV}$. The absorption spectra and emission spectra were recorded with a UV2550 scanning spectrophotometer (Shimadzu, Japan) and an F-2500 Spectrofluorometer (Hitachi, Japan), respectively. To measure their photostability in physiological environment and weak acidic environment, our as-prepared QDs and a similar commercial CdTe QDs 
were dissolved in deionized ultra-pure water with a $\mathrm{pH}$ value adjusted to 7.4 or 5.0 with $\mathrm{NaOH}$ or $\mathrm{HCl}$, respectively. Then the QD solutions were added into the microplate and their mean fluorescence intensities (MFI) at peak emission were measured at $405 \mathrm{~nm}$ excitation and $599 \mathrm{~nm}$ emission every 20 minutes for $15 \mathrm{~h}$ periods with a fluorescence microplate reader (Flexstation 3, Molecular Device). To determine the composition of CdTe QDs, the concentration of mercaptosuccinate capping ligands was determined by measuring the total organic carbon (TOC, TOC-VCPH, Shimadzu, Japan). The concentration of cadmium from QDs was measured by graphite furnace atomic absorption spectroscopy (GFAA, AA800 Spectrometer, Thermo scientific). To measure the free cadmium ions released from CdTe QDs, $2 \mathrm{~mL}$ CdTe QDs stock solution (1 mg/mL) was added into a dialysis tube (molecular-weight cutoff of 400 daltons, MWCO-400, 3M Co. Ltd.) and then placed in polypropylene beakers containing $500 \mathrm{~mL}$ ultra pure water. The dialysis experiment lasted for $24 \mathrm{~h}$, and $2 \mathrm{~mL}$ was taken at the $6^{\text {th }}, 18^{\text {th }}$ and $30^{\text {th }}$ hour. Finally, the collected sample was analyzed using AAS. All the measurements were performed at room temperature and in triplicate.

\subsection{Cell culture and treatments}

Primary HUVECs were obtained from human umbilical cord veins according to Ling et a1. (Ling et al. 2003). In brief, the vein was cannulated and filled with $0.1 \%$ type I collagenase. After incubation $\left(37^{\circ} \mathrm{C}, 15 \mathrm{~min}\right)$, the obtained pellets were centrifugated and resuspened in Medium200 with LSGS at a concentration of $10^{5}$ cells/mL. The cells were used at passage 2 to 4 in our study. After reaching $90 \%$ 
confluence, M200 with LSGS was removed and then HUVECs were cultured with M200 (containing 2\% FBS) in the presence of CdTe QDs at different final concentrations (range from 0.1 to $100 \mu \mathrm{g} / \mathrm{mL}$ ) for 12 or $24 \mathrm{~h}$. Untreated control cells were incubated with medium (containing only $2 \%$ FBS) alone.

\subsection{Cell viability assessment}

The viability of HUVECs treated with CdTe QDs was estimated with both 3-(4,5-dimethylthiazolyl-2)-2, 5-diphenyltetrazolium bromide (MTT) (Andersson et al. 2009) and calcein ester green fluorescence assays. For the MTT assay, after reaching 90\% confluence, M200 with LSGS was removed and then HUVECs in 96-well culture plates were cultured with M200 (containing 2\% FBS) in the presence of CdTe QDs at a concentration gradient of 0.1 to $100 \mu \mathrm{g} / \mathrm{mL}$ for $24 \mathrm{~h}$. Then, the culture medium was removed; $150 \mu \mathrm{L}$ MTT solution $(0.5 \mathrm{mg} / \mathrm{mL})$ was added to each well and incubated for another $4 \mathrm{~h}$. The amount of viable cells in each well was determined by the absorbance of solubilized formazan. The optical density (O.D.) was measured at $490 \mathrm{~nm}$ with a microplate reader (Versa Max, Molecular Devices). For using a calcein ester green fluorescence assay, cells were washed with PBS twice after treatment with QDs, and incubated with $2 \mu \mathrm{M}$ calcein-AM for $10 \mathrm{~min}$. The fluorescence images of cells were obtained with a laser scanning confocal microscope (LSM 510, Carl Zeiss).

\subsection{Detection of cellular apoptosis}

Exposed membrane phosphatidylserine (PS) in apoptotic cells induced by QDs was identified by flow cytometry (FCM) using a commercial apoptosis detection kit (Vybrant ${ }^{\circledR}$ Apoptosis Assay Kit, Invitrogen), according to Hsiao et al. (Hsiao et al. 
2009). In brief, after 90\% confluence, HUVECs were cultured with M200 (containing 2\% FBS) in the presence of 0.1 to $10 \mu \mathrm{g} / \mathrm{mL}$ CdTe QDs for $24 \mathrm{~h}$. The treated cells cells $\left(1 \times 10^{5}\right)$ were rinsed twice with PBS, harvested by trypsinization and labeled with 5 $\mu \mathrm{g} / \mathrm{mL}$ FITC-conjugated Annexin- $\mathrm{V}$ for $15 \mathrm{~min}$. The fluorescence of Annexin-V-positive HUVECs was measured with FACScalibur flow cytometer (Becton Dickinson, USA) on the FL-1 detector (530 $\pm 15 \mathrm{~nm}$, band pass). For statistical significance, at least 10,000 cells were analyzed in each sample.

The involvement of ROS in CdTe QDs-induced apoptosis was also examined by this FCM assay. In brief, HUVECs were pre-incubated with an antioxidant and ROS scavenger $\mathrm{N}$-acetylcysteine (NAC, $1 \mathrm{mM}$ ) for $2 \mathrm{~h}$ and then with a QDs suspension (10 $\mu \mathrm{g} / \mathrm{mL}$ ) for an additional $24 \mathrm{~h}$. To study whether CdTe QDs-induced apoptosis in HUVECs was caspase-dependent, caspase inhibitors Z-VAD-FMK (50 $\mu \mathrm{M})$ or Ac-DEVD-CHO (20 mM) were incubated with HUVECs for $1 \mathrm{~h}$ prior to the addition of QDs (10 $\mu \mathrm{g} / \mathrm{mL})$. Then, after treatment, the apoptotic HUVECs were also detected on the FL-1 detector (530 $\pm 15 \mathrm{~nm}$, band pass) of the FACScalibur flow cytometer.

\subsection{Evaluation of intercellular ROS and mitochondrial membrane potential} $\left(\Delta \psi_{m}\right)$

DCFH-DA was used for ROS detection. DCFH-DA is transported across the cell membrane and cleaved by nonspecific esterases to form DCFH, which is further oxidized by ROS to form the fluorescent compound DCF. HUVECs were rinsed twice with PBS, trypsinized and stained with DCFH-DA $(5 \mu \mathrm{M})$ after 12 or 24 h QDs treatment. The green fluorescence of DCF was detected on the FL1 detectors in a 
FACScalibur flow cytometer. HUVECs stimulated with Rosup (50 $\mu \mathrm{g} / \mathrm{mL})$ for $30 \mathrm{~min}$ were taken as positive controls of ROS generation.

For quantitative analysis of $\Delta \psi_{m}$, QDs-treated HUVECs were also rinsed twice with PBS, trypsinized and then stained with JC-1. JC-1 is a lipophilic probe which potential-dependently accumulated in mitochondria and its fluorescence emission shifts from red ( $\sim 590 \mathrm{~nm}$, J-aggregates) to green ( $\sim 525 \mathrm{~nm}$, J-monomers) when $\Delta \psi_{m}$ decreases. The fluorescence of J-monomers was quantified on FL1 detector of FACScalibur flow cytometer. The negative control for flow cytometer compensation was prepared by addition of $\Delta \psi_{m}$ disrupter m-chlorophenyl-hydrazone (CCCP, $50 \mu \mathrm{M}$ ) to untreated HUVECs for the complete mitochondrial depolarization. To further clarify the role of QDs-caused ROS in induction of endothelial depolarization of mitochondria, HUVECs were pre-incubated with NAC $(1 \mathrm{mM})$ for $2 \mathrm{~h}$ and then with a QDs suspension (10 $\mu \mathrm{g} / \mathrm{mL})$ for an additional $24 \mathrm{~h}$. Subsequently, the cells were trypsinized, loaded with JC-1 and analyzed by flow cytometer. For statistical significance, at least 10,000 cells were analyzed in each sample.

\subsection{Visualization of mitochondria structure}

To examine mitochondrial structure in HUVECs treated with QDs for $24 \mathrm{~h}$, MitoTracker Red FM (1 $\mu \mathrm{M})$ was added after twice PBS washing. After a short incubation period of 15 minutes, the fluorescence images of mitochondria were obtained using a LSM510 laser scanning confocal microscope (Carl Zeiss, Germany).

\subsection{Cytochrome $c$ immunostaining in QDs treated HUVECs}

The release of cytochrome $c$ from mitochondria was detected using 
immunostaining analysis. Briefly, HUVECs were washed twice with PBS and loaded with $100 \mu \mathrm{M}$ MitoTracker Red FM for 20 minutes after 24 h QDs treatment. Then, cells were fixed with 3.7\% paraformaldehyde and permeabilized for immunostaining. Mouse polyclonal anti-cytochrome $c(1: 200)$ and FITC-conjugated mouse IgG (1:500) were used. The translocation of cytochrome $c$ from mitochondria to cytoplasm was observed by a Carl-Zeiss LSM 510 confocal laser microscope.

\subsection{Immunoblot analysis of cellular expression of apoptotic proteins}

HUVECs exposed to CdTe QDs for 24 h were rinsed with cold PBS and lysed for $30 \mathrm{~min}$ in cold radioimmune precioitation assay (RIPA) buffer (1\% Triton X-100, 50 mM Tris/HCl, pH 7.4, 300 mM NaCl, $10 \mu \mathrm{g} / \mathrm{mL}$ aprotinin, $10 \mu \mathrm{g} / \mathrm{mL}$ leupeptin, $1 \mathrm{mM}$ sodium orthovanadate, and $2 \mathrm{mg} / \mathrm{mL}$ iodoacetamide). After removing cellular debris by centrifugation $\left(13,000 \mathrm{rpm}, 10 \mathrm{~min}, 4^{\circ} \mathrm{C}\right)$, the cell lysates were subjected to $12 \%$ sodium dodecyl sulfate polyacrylamide gel electrophoresis (SDS-PAGE) and subsequently transferred onto polyvinylidene difluoride (PVDF) membranes (Millipore). Blocking of unspecific binding sites with nonfat dry milk (5\% in Tris-buffered saline containing $0.1 \%$ Tween-20) was followed by incubation with primary antibodies (Bcl-2 1:500; Bax 1:1000; caspase-9 1:500; caspase-3 1:1000), which were detected with horseradish peroxidase (HRP)-conjugated secondary antibodies, using a TMB Liquid Substrate kit.

\subsection{Statistical analysis}

Experiments were performed at least three times. Data were presented as mean \pm SD and evaluated for statistical significance by one- or two-way analysis of variance, 
followed by a post hoc test.

\section{Results}

\subsection{Characterizations of CdTe QDs}

HRTEM images (Fig.1A) showed that the CdTe QDs as-prepared have a highly crystalline nature and a very uniform size ( $~ 4 \mathrm{~nm})$. The typical visible absorption and photoluminescence (PL) spectra of CdTe QDs in Fig.1B showed that the QDs has a narrow, symmetric emission spectra (about $60 \mathrm{~nm}$ full width at half maximum, FWHM) and the emission peak at $599 \mathrm{~nm}$. According to Peng et al. (Yu et al. 2003), we calculated the diameter and concentration of QDs from the UV-visible absorption spectra. For the as-prepared CdTe QDs, the diameter of QDs was about $3.36 \mathrm{~nm}$ and the molarity of $10 \mu \mathrm{g} / \mathrm{mL}$ stock solution was about $22.7 \mathrm{nM}$.

In this study, we used MSA as the surface coating to protect the QDs core. The concentration of mercaptosuccinate capping ligands was assessed by measuring the TOC. The TOC content in $10 \mu \mathrm{g} / \mathrm{mL}$ QDs stock solution was about $0.686 \pm 0.03$ $\mu \mathrm{g} / \mathrm{mL}$. Because the TOC in mercaptosuccinate $\left(\mathrm{C}_{4} \mathrm{H}_{5} \mathrm{O}_{4} \mathrm{~S}^{-}\right)$was about $32 \%$ of the total mass, the content of MSA can be calculated as $2.14 \pm 0.03 \mu \mathrm{g} / \mathrm{mL}$. On the other hand, the concentration of Cd from the $10 \mu \mathrm{g} / \mathrm{mL}$ QDs stock solution was determined as $5.77 \pm 0.16 \mu \mathrm{g} / \mathrm{mL}$ by AAS. Based on these above results, the formula $\mathrm{Cd}_{10} \mathrm{Te}_{3.18}\left(\mathrm{SCH}_{2} \mathrm{CHCOOHCOOH}\right)_{2.78}$ is suggested. Respecting the instrument error and the surface-to-volume atom ratio (Peng and Peng 2002; Yu et al. 2003), our

results indicated that the CdTe core QDs were well capped with MSA, which was 
alternatively confirmed by the free cadmium ion assays (Fig.2A). After dialysis of 1 $\mathrm{mg} / \mathrm{mL}$ CdTe QDs for $30 \mathrm{~h}$, the $\mathrm{Cd}^{2+}$ in the dialysis buffer solution was only $15.99 \pm$ $1.30 \mathrm{ng} / \mathrm{mL}$, suggesting there was only a little $\mathrm{Cd}^{2+}$ leakage from the MSA-capped CdTe QDs. Furthermore, Fig.2B showed that the MFI at peak emission of MSA-capped CdTe QDs under a weak acidic condition $(\mathrm{pH}=5)$ was slightly lower than that under a physiological condition $(\mathrm{pH}=7.4)$ through the tested period. But the commercial CdTe QDs, which was capped with unknown micromolecule organic acid, appears to completely lose its fluorescence when the $\mathrm{pH}$ value was adjusted to 5 . Hence, our MSA-capped CdTe QDs have favorable properties of being water-soluble and highly $\mathrm{pH}$ stable, and present promising prospects in the biomedical applications.

\subsection{CdTe QDs decreases cell viability of HUVECs in a dose-dependent manner}

To examine the direct toxic effects on vascular ECs, cultured HUVECs were treated with CdTe QDs for 24 h. As shown in Fig.3A, CdTe QDs dose-dependently decreased the cell viability of HUVECs, as a gradual decline in cellular calcein fluorescence was observed in the presence of $0.1 \sim 10 \mu \mathrm{g} / \mathrm{mL}$ CdTe QDs. MTT assay showed that significant cell death was observed with CdTe QDs at a concentration of $1 \mu \mathrm{g} / \mathrm{mL}$ and above (Fig.3B, $p<0.01$, one-way ANOVA). The $\mathrm{TC}_{50}$ value (concentration of CdTe QDs causes 50\% cell death) was found at $10.60 \mu \mathrm{g} / \mathrm{mL}$ (about $24.06 \mathrm{nM}$ ) determined by log-logit transformation, indicating the remarkable endothelial toxicity of CdTe QDs.

\subsection{CdTe QDs lead to apoptotic cell death of HUVECs}

To further assess the extent of QDs-induced injuries, we detected the apoptosis in 
QDs-treated HUVECs by flow cytometry, using an Annexin-V apoptosis detection kit. The translocation of PS from the inter leaflet of the plasma membrane to outer leaflet, and then can be labeled by Annexin- $\mathrm{V}$, is one of the typical features of cells undergoing apoptosis process. After $24 \mathrm{~h}$ incubation with QDs, the percentages of Annexin-V-positive HUVECs (Fig.4) increased gradually depending on CdTe QDs concentration ( $4.48 \pm 1.17 \%$ at control, $4.73 \pm 0.81 \%$ at $0.1 \mu \mathrm{g} / \mathrm{mL}, 6.37 \pm 0.35 \%$ at 1 $\mu \mathrm{g} / \mathrm{mL}$ and $22.49 \pm 1.34 \%$ at $10 \mu \mathrm{g} / \mathrm{mL}, p<0.01$, one-way ANOVA). These results suggested that CdTe QDs could induce rapid apoptosis of HUVECs, and exerted direct pro-apoptotic effects in a dose-dependent manner.

\subsection{CdTe QDs elevate intracellular ROS levels and subsequent mitochondrial} dysfunction in HUVECs

The effect of QDs on ROS generation in HUVECs was assessed by measuring the oxidation of DCFH-DA to DCF using flow cytometer. The exposure of HUVECs to CdTe QDs for $12 \mathrm{~h}$ resulted in a dose-dependent increase of intracellular ROS (Fig.5B). Compared with control, 0.1, 1 and $10 \mu \mathrm{g} / \mathrm{mL}$ CdTe QDs significantly promoted ROS generation by $77.76 \%, 100.97 \%$ and $119.35 \%$ in HUVECs, respectively ( $p<0.05$, one-way ANOVA). However, the intracellular ROS content was gradually increased to $138.10 \%$ of control at $0.1 \mu \mathrm{g} / \mathrm{mL}$ and $135.63 \%$ of control at 1 $\mu \mathrm{g} / \mathrm{mL}$ after $24 \mathrm{~h}$ incubation, and it even restored to $2.63 \%$ lower than the control value at $10 \mu \mathrm{g} / \mathrm{mL}$ group (Fig.5B). These results revealed that CdTe QDs-induced elevation of intracellular ROS production may be earlier than the apoptosis, suggesting ROS might play a role in CdTe QDs-induced apoptosis of HUVECs. 
Since oxidative stress is known to impair mitochondria, whose functions are essential for maintaining cellular homeostasis, we studied the effect of CdTe QDs on $\Delta \psi_{m}$ and the morphology of mitochondria in HUVECs. The flow cytometric analysis (Fig.6B) showed that incubation of HUVECs with $0.1 \mu \mathrm{g} / \mathrm{mL}$ CdTe QDs had no visible effect on cellular $\Delta \psi_{m}$. However, a higher concentration of CdTe QDs caused a pronounced reduction of $\Delta \psi_{m}$, as the percentage of unhealthy HUVECs with low $\Delta \psi_{m}$ was remarkably increased by $89.77 \%$ or $166.41 \%$ in the presence of 1 or $10 \mu \mathrm{g} / \mathrm{mL}$ CdTe QDs, respectively ( $p<0.05$, one-way ANOVA). In addition, the CdTe QDs induced cytotoxic morphological changes of mitochondria in HUVECs (Fig.6C), converting the mitochondrial tubular shape to a spherical conformation after $24 \mathrm{~h}$ exposure to $10 \mu \mathrm{g} / \mathrm{mL}$ CdTe QDs. These results suggested that CdTe QDs could induce severe impairments of mitochondria including its fragmentation and depolarization.

Moreover, we use the ROS scavenger NAC to clarify the role of ROS in CdTe QDs-induced mitochondrial impairment and apoptosis in HUVECs. As shown in Fig.7A, NAC could effectively protect HUVECs from CdTe QDs-induced disruption of $\Delta \psi_{m}$; the ratio of HUVECs with low $\Delta \psi_{m}$ reduced by $44.69 \%$ in the presence of 1 mM NAC. The FCM data (Fig.7B) also confirmed that NAC could markedly inhibits the CdTe QDs-induced endothelial apoptosis; the percentage of apoptosis in both QDs and NAC co-treated HUVECs was reduced by 55.36\% than that in QDs group. Altogether, these data indicated that QDs-caused intracellular ROS elevation was directly involved not only in the QDs-induced mitochondrial injury but also in 
QDs-induced apoptosis.

\subsection{CdTe QDs activated the mitochondrial apoptosis pathways in HUVECs}

Besides maintaining cellular metabolism, mitochondria have also been shown to fulfill very important functions in the signal transduction for apoptosis. Therefore, to further investigate the molecular mechanism of CdTe QDs-induced HUVECs apoptosis, we detected the expression of mitochondrial apoptosis regulatory proteins by immunoblotting analysis. The expression level of the pro-survival Bcl-2 in HUVECs dramatically decreased, whereas the level of the pro-apoptotic Bax significantly increased after $24 \mathrm{~h}$ of treatment (Fig. 9A). Because Bax is involved in release of cytochrome $c$, which subsequently triggers activation of caspase proteases and death of a cell by apoptosis, we then examined the subcellular distribution of cytochrome $c$ by immunofluorescence. Cytochrome $c$, as a component of the electron transport chain, is found loosely associated with the inner membrane of the mitochondrion. Indeed, in control cells, the cytochrome $c$ (green, Fig.8A) showed punctuate staining in the cytoplasm/perinuclear region, and it was well co-localized with the mitochondria (red, Fig.8B and C). In contrast, when the HUVECs treated with $10 \mu \mathrm{g} / \mathrm{mL}$ CdTe QDs for $24 \mathrm{~h}$, the staining for cytochrome $c$ in those cells was not punctate and perinuclear but, rather, diffuse (Fig.7E). There was scarce overlay with mitochondria can be observed in QDs-treated HUVECs (Fig.8D and F), suggesting that the bulk of cytochrome c leaked from endothelial mitochondria after QDs treatment. Collectively, these results indicated that the protein expression of pro-apoptotic molecules increased in CdTe QDs-treated HUVECs. 
The initiator caspase-9 and effector caspase-3, which play vital roles in the mitochondrial-dependent apoptotic pathway of cells, were both activated in HUVECs following treatment with CdTe QDs, because the immumoblotting analysis showed that the cleavage of both caspase- 9 and caspase- 3 could be detected after 24 h CdTe QDs treatment (Fig.9B). The pan-caspase inhibitor Z-VAD-FMK and specific caspase-3 inhibitors Ac-DEVD-CHO were used to analyze the involvement of caspases in CdTe QDs-induced apoptosis in HUVECs. As shown in Fig. 8B, the pre-treatment of HUVECs with either Z-VAD-FMK or Ac-DEVD-CHO significantly decreased CdTe QDs-induced apoptosis to $7.48 \pm 1.03 \%$ and $13.07 \pm 0.98 \%$. Taken together, our results showed that CdTe QDs directly activated the intrinsic mitochondrial apoptosis pathway in HUVECs, and this apoptotic process was clearly caspase-dependent.

\section{Discussion}

The present study demonstrated that MSA-capped CdTe QDs directly caused cytotoxic injury of human vascular ECs. Treatment HUVECs with the CdTe QDs significantly elevated intracellular ROS, induced widespread mitochondrial dysfunction, activated the mitochondrial apoptosis pathway, and ultimately triggered apoptotic cell death in HUVECs. These results provided the first demonstration of the direct effects of QDs on vascular ECs as well as strong evidences for molecular mechanisms of QDs-induced endothelial cytotoxicity.

Semiconductor QDs have attracted great concern as novel fluorophores for 
labeling cells or tissues and drug tracing during the last decade (Jaiswal et al. 2003; Medintz et al. 2005). CdTe QDs, with narrow band gaps and wide Bohr exciton radius, have smaller sizes than most of commercial CdSe QDs at the red or near infrared (NIR) range, which enable they can be effectively resolved over the same size range. Besides that, our data of element composition determination and $\mathrm{Cd}^{2+}$ leakage assay confirmed that our CdTe core QDs was well capped with MSA, that was reported to provide more excellent stabilities of QDs against aggregation at low $\mathrm{pH}$ than mostly employed mercaptoacetic acid (MPA) or dihydrolipoic acid (DHLA) (Algar and Krull 2007). In fact, our as-prepared QDs have efficient luminescence, and they do have higher pH stability (Fig.2B) than that of a similar commercial QDs. Hence, our QDs with excellent optical properties are suitable for biomedical applications such as bioimaging, composite drug delivery or disease diagnosis agents. However, little is known whether QDs affect endothelial function when they enter vasculature through injection as the imaging agents. Therefore, employing HUVECs as an in vitro model, we explored the potential endothelial toxicity of these MSA-capped CdTe QDs.

In this study, we tested the endothelial toxicity of MSA-capped CdTe QDs in the concentration range from 0.1 to $100 \mu \mathrm{g} / \mathrm{mL}$ (coresp. molarity was about $0.227 \mathrm{nM}$ to $227 \mathrm{nM}$ ). According to previous reports, the concentration which we tested in our following experiments is similar to the doses that have actually used in in vivo bioimaging (up to hundreds of nanomole) (Ballou et al. 2007; Ballou et al. 2004; Cai et al. 2006; Gao et al. 2004; Jayagopal et al. 2007; Larson et al. 2003; Tada et al. 2007) or tested in other in vitro toxicological studies (up to hundreds of nanomole or $\mu \mathrm{g} / \mathrm{mL}$ ) 
(Chang et al. 2009; Chen et al. 2010; Choi et al. 2007; Lovric et al. 2005; Su et al. 2009; Su et al. 2010; Tang et al. 2008; Wu et al. 2010). Then, our cell viability assays (Fig. 3) revealed that CdTe QDs caused significant cell loss in a dose-dependent manner (at or above $0.1 \mu \mathrm{g} / \mathrm{mL}$ ), demonstrating CdTe QDs manifested cytotoxic effect against HUVECs. Quantitive cytometric analysis disclosed that, in HUVECs, exposure to CdTe QDs induced dose-dependent apoptotic cell death (Fig.4), which might compromise vasoregulation, increase smooth muscle cells (SMCs) proliferation and/or migration and facilitate blood clotting (Bombeli et al. 1997; Busse et al. 1985; Raymond et al. 2004), leading to CVDs such as atherosclerosis and thrombus. Notably, the concentration of CdTe QDs required to promote endothelial toxicity $\left(\mathrm{TC}_{50}\right)$ in our study was much lower than most of that in other cell types (Chen et al. 2010; Choi et al. 2007,(Su et al. 2010), (Su et al. 2009; Wu et al. 2010), (Chang et al. 2009; Chen et al. 2010; Choi et al. 2007; Lovric et al. 2005; Shiohara et al. 2004; Tang et al. 2008). A possible explanation for this difference is that the tested cell types have different cellular sensitivity to QDs. In other words, vascular ECs are more susceptible to damage by QDs.

QDs were reported to generate reactive singlet oxygen species (Samia et al. 2003). To explore if QD could induced overabundant intracellular ROS and if that was tightly associated with their endothelial toxicity (Cho et al. 2007), we measured the ROS intensity and potential injuries of mitochondrial functions in QDs-treated HUVECs. Here we observed that QDs treatment could trigger a twofold elevation of intracellular ROS (Fig. 5), followed by obvious disruption of $\Delta \psi_{m}$ (Fig.6B), which 
could be inhibited by ROS scanvenager NAC (Fig.7), as well as mitochondrial fragmentation (Fig.6C). Hence, considering the free $\mathrm{Cd}^{2+}$ released from MSA-capped CdTe QDs is little (Fig.2A), we suppose that QDs-induced endothelial toxicity was mostly mediated by elevated intracellular ROS; and mitochondria may be a major target organelle. This undesirable oxidative damage caused by QDs also reminder that the surface modification of QDs, which may reduce the production of ROS, is of crucial importance in order to prepare non-toxic fluorescent probes for future scientific and clinical usage. Meanwhile, unlike other cells, vascular ECs obtain most of their energy from anaerobic glycolysis, and the collapse of endothelial $\Delta \psi_{m}$ inhibits mitochondrial production of NO, which can rapidly modulate cellular functions and apoptosis (Culic et al. 1997; Dedkova et al. 2004; Quintero et al. 2006). Therefore, QDs-induced mitochondrial oxidative damage in ECs may represent an important step in the development of endothelial dysfunction per se rather than a key role in diminishment of cellular energy production in other cells.

On the other hand, nanomaterials-generated high level of ROS was also confirmed to lead detrimental autophagic cell death as well as necrosis (Liu and Sun 2010; Stern et al. 2008; Yamawaki and Iwai 2006). Our preliminary results (data not shown) revealed that $10 \mu \mathrm{g} / \mathrm{mL}$ CdTe QDs could convert the cytosolic protein microtubule-associated protein 1 light chain 3-I (LC3-I) to LC3-II which was associated with the formation of autophagosomes. Combing the previous reports and our data, we conjectured that autophagic and/or necrotic cell death were possibly implicated in QDs-indcued endothelial damage and this could partially explain the 
seemingly contradictory data that the percentage of apoptotic HUVECs treated with $10 \mu \mathrm{g} / \mathrm{mL}$ CdTe QDs (a dose similar to the $\mathrm{TC}_{50}$ value determined by MTT assay) was lower than 50\%. Nevertheless, the question of whether QDs directly induce autophagy or necrosis in ECs requires further investigation.

Furthermore, the present study found that QDs directly activated the intrinsic mitochondrial apoptosis pathway, which is widely involved in vascular diseases (Davidson and Duchen 2007; Tanaka et al. 2003; Vindis et al. 2005). After 24 h incubation, CdTe QDs caused an increase of pro-apoptotic Bax but a decrease of pro-survival Bcl-2 (Fig.9A); and they also caused the release of cytochrome $c$ (Fig.8), accompanied by the cleavage of caspase-9 and caspase-3 (Fig.9A). Moreover, the apoptosis caused by CdTe QDs was significantly reduced by caspase inhibitors (Fig.9B), indicating caspases are essential for CdTe QDs-induced apoptosis in ECs. It is noteworthy that our result is different from the previous study by Lovrić et al., in which they observed that QDs-induced cell death was caspase-independent in cells with caspase-3 expression (PC12 cells) or without (MCF-7 cell line) (Lovric et al. 2005b). As the QDs used in two studies were of similar composition, the same dose (10 $\mu \mathrm{g} / \mathrm{mL})$ and induced similar impairments of mitochondria in all three cell types, the discrepancy of downstream apoptosis signal components of mitochondria seemed to arise from different cell types. Collectively, the understanding of the mechanism underlying QDs-induced cell death is still far from complete, and may be complicated by cell types and signaling context differences.

In conclusion, we have demonstrated for the first time that QDs increased the 
generation of intracellular ROS, impaired mitochondrial functions, activated the mitochondrial apoptosis pathway and finally promoted apoptotic cell death in HUVECs. Our overall findings provided strong evidence that the QDs circulating in blood was a potential significant risk factor for CVDs. With increased utilization of QDs in biomedical research as well as the potential clinical usage, these data also offer helpful guidance on future safe use of QDs and help researchers improve the manipulation of QDs to make them more suitable tools in life science.

\section{Conflicts of interest}

The authors have no conflict of interest.

\section{Acknowledgement}

The authors acknowledge the assistance of Wanting Niu and Wei Cai for the AAS and TOC measurements. We would also like to thank Dr. Chuxiong Hu for his kind help during this study. 

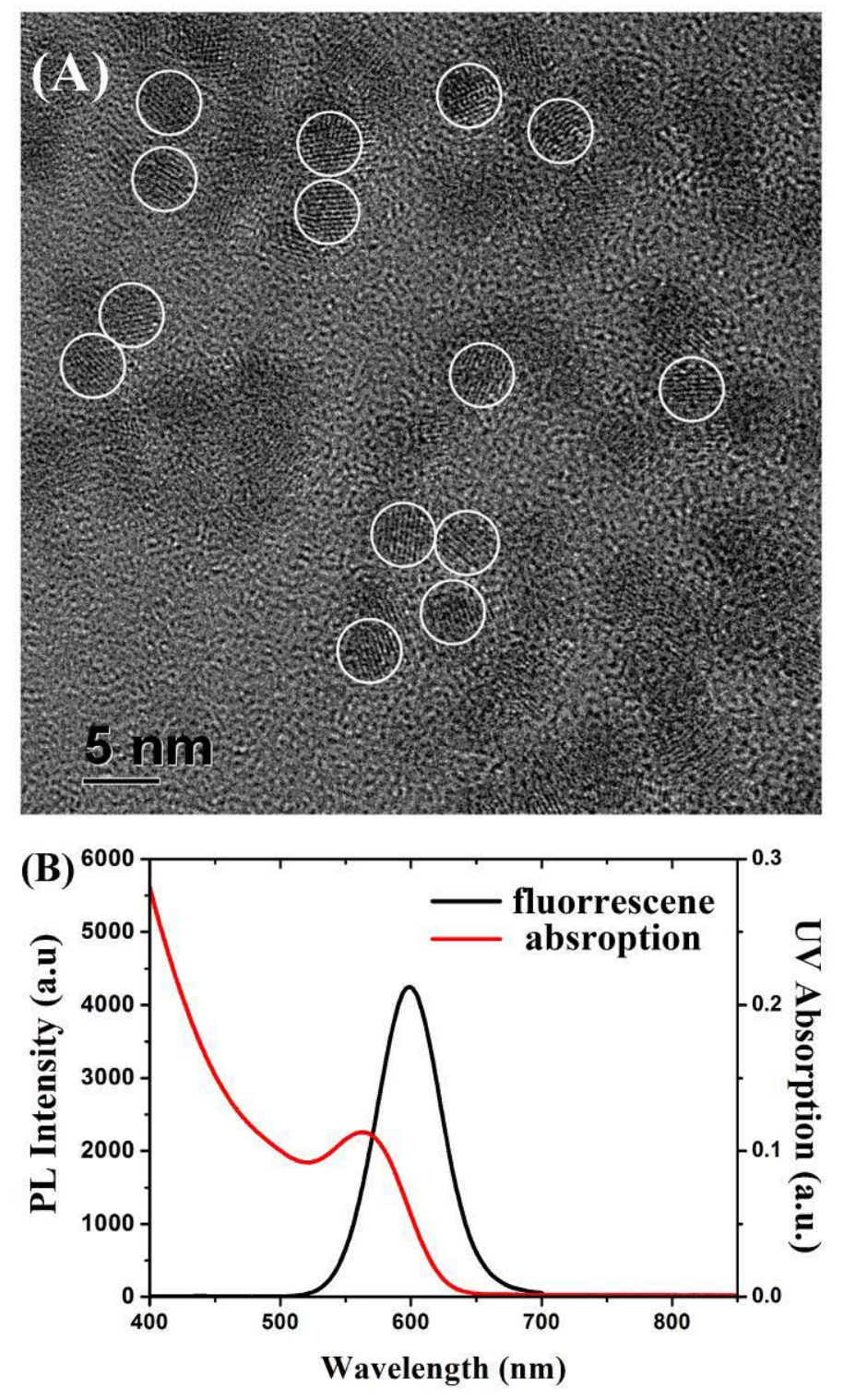

Fig.1 MSA-capped CdTe QDs characterizations. (A) HRTEM of CdTe QDs; (B) Absorption and PL spectra of CdTe QDs. Bar = $5 \mathrm{~nm}$. 
(A)
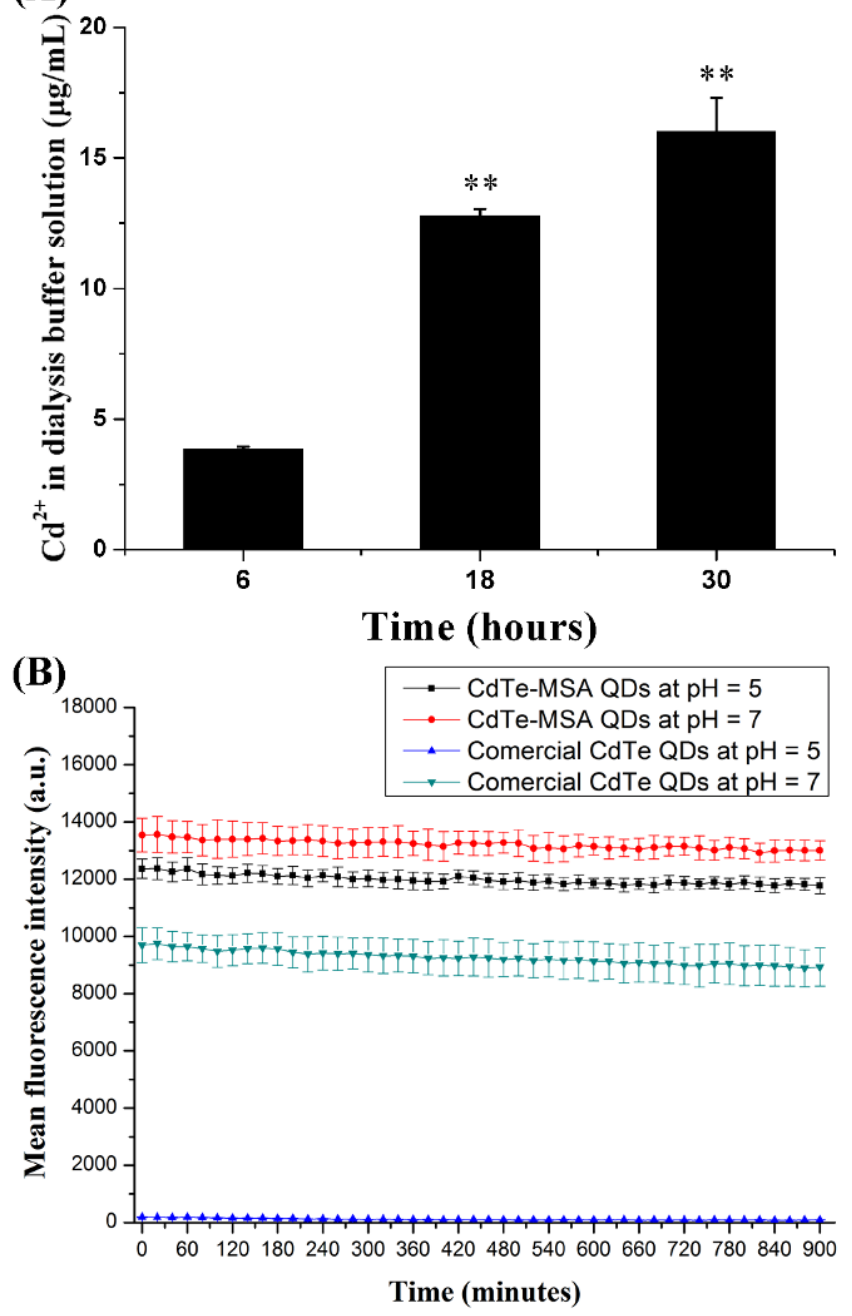

Fig.2 MSA-capped CdTe QDs characterizations. (A) The concentration of free $\mathrm{Cd}^{2+}$ in the dialysis buffer solution; (B)Fluorescence stability of CdTe QDs were measured at a concentration of $100 \mu \mathrm{g} / \mathrm{mL}$ in PBS buffer adjusted to different $\mathrm{pH}$ values (mean $\pm \mathrm{SD}, \mathrm{n}=6$ ). 

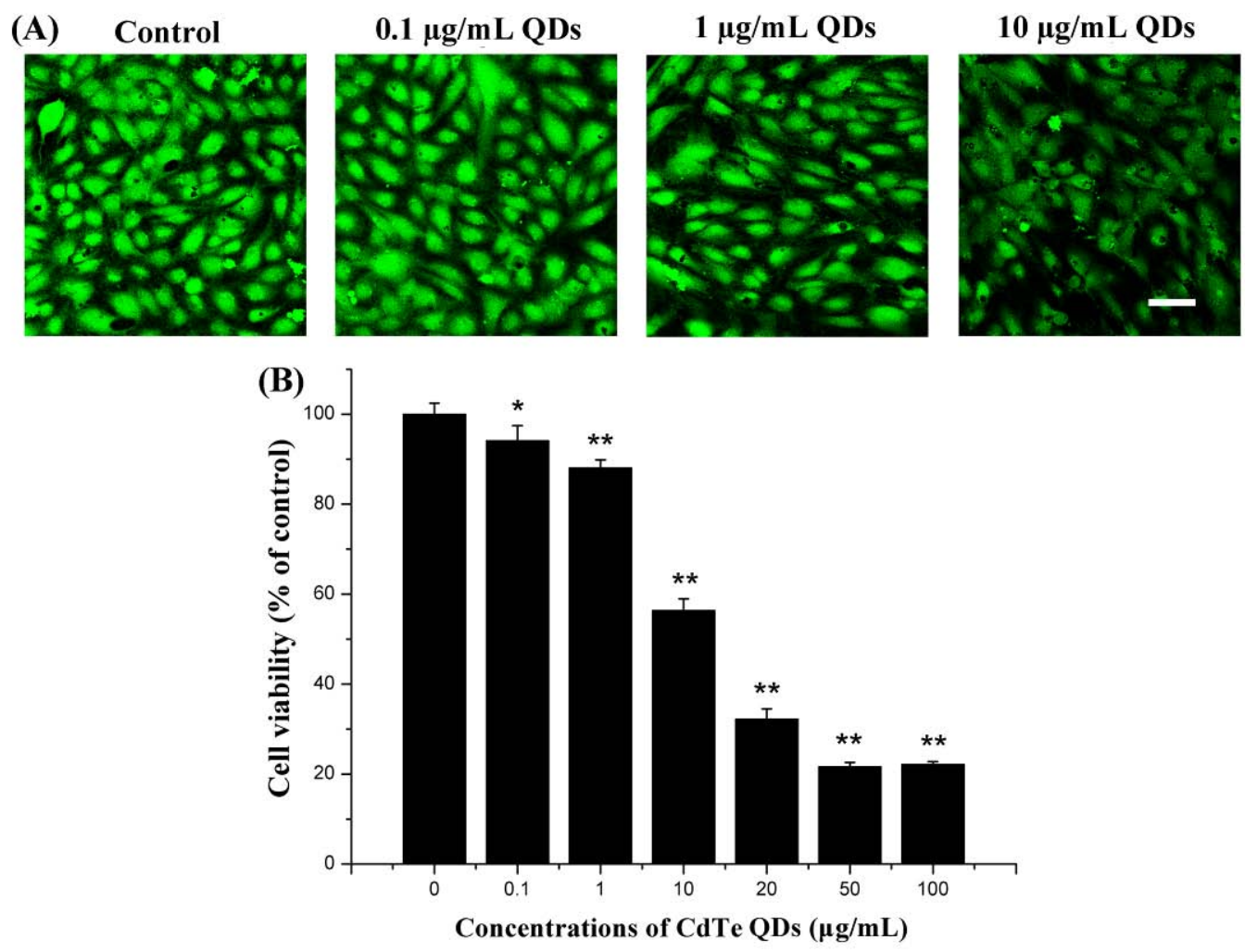

Fig.3 CdTe QDs caused decline of cell viability in HUVECs. HUVECs were treated with various concentrations of QDs for $24 \mathrm{~h}$. Cell viability measured by (A) Calcein-AM fluorescence staining; and (B) MTT assay. Data represent mean \pm SD of four determinations. ${ }^{*} p<0.05$ and ${ }^{* *} p<0.01$ compared with control. Bars $=50 \mu \mathrm{m}$ 

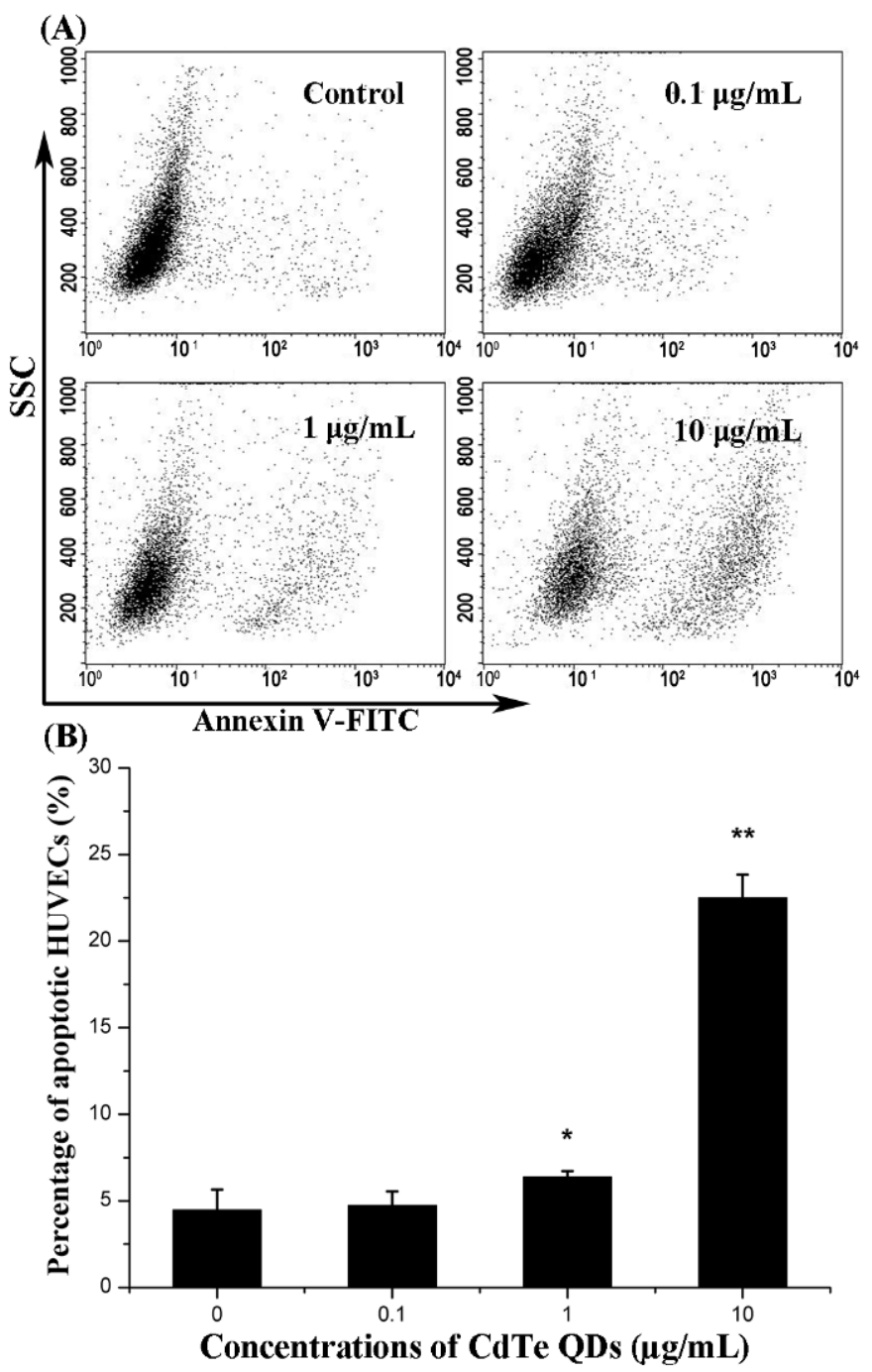

Fig.4 CdTe QDs caused apoptosis of HUVECs. (A) PS exposure resulting from 24 h CdTe QDs treatment, HUVECs were stained with FITC conjugated Annexin-V and subjected to cytoflurometric analysis. (B) Quantitation of data obtained in (A). Data represent mean \pm SD of four determinations. * $p<0.05$ and ${ }^{* *} p<0.01$ compared with control. 

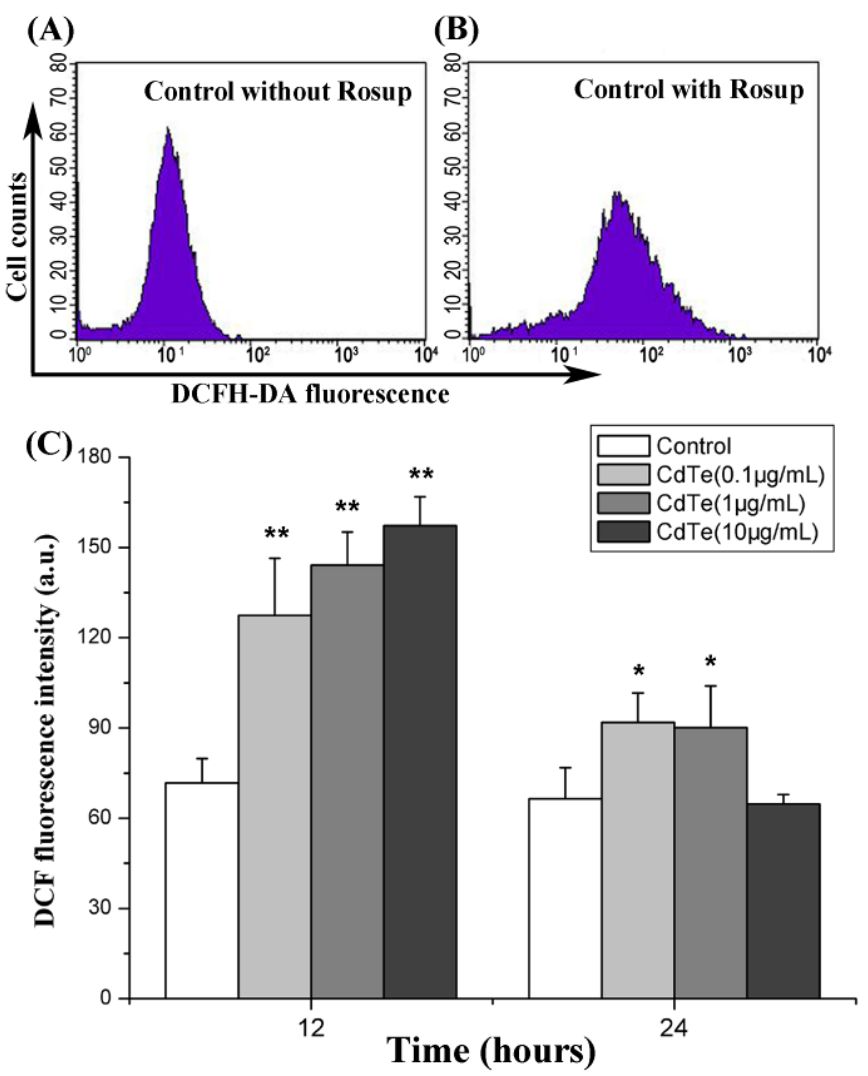

Fig.5 Effect of CdTe QDs on ROS levels in HUVECs. (A) HUVECs stimulated with Rosup ( $50 \mu \mathrm{g} / \mathrm{mL}$ ) for 30 min were taken as positive control; (B) The DCF mean fluorescence intensity of HUVECs treated with or without CdTe QDs were determined by flow cytometer. Data represent mean \pm SD of six determinations. ${ }^{*} p<0.05$ and $* * p<0.01$ compared with control. 


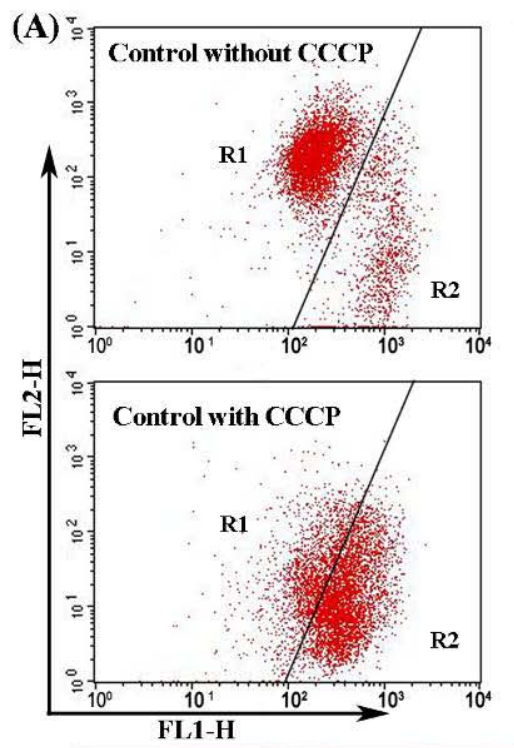

(B)

(C)

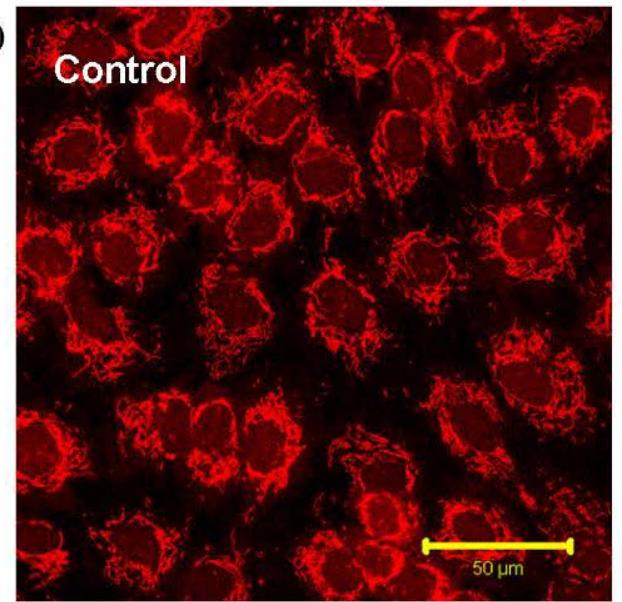

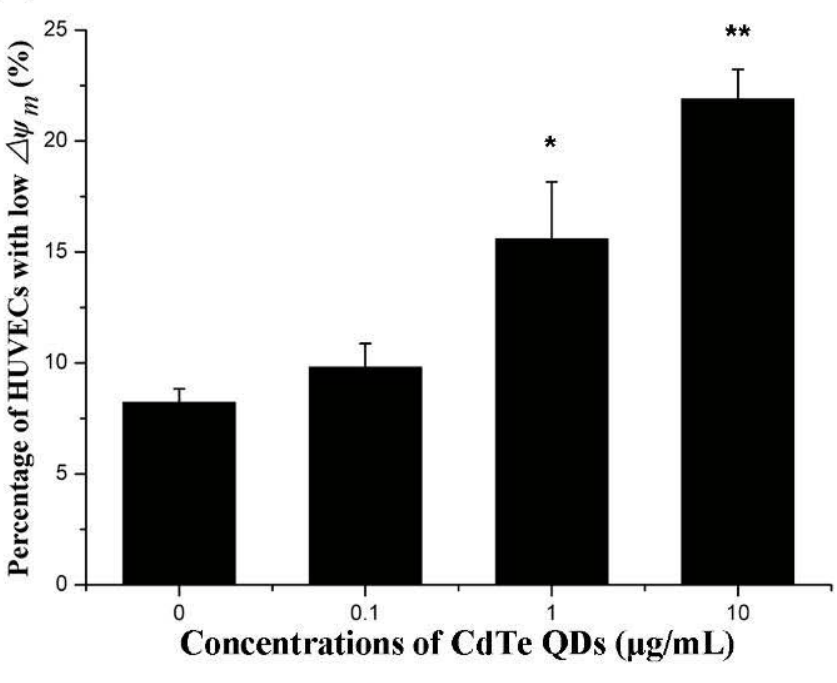

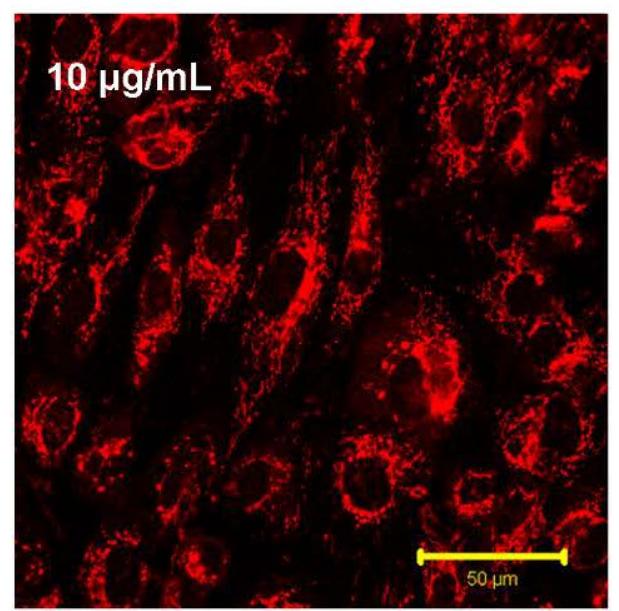

Fig.6 Effect of CdTe QDs on mitochondrial function in HUVECs. (A) Increase of the fluorescence of J-monomers after addition of CCCP to untreated HUVECs which were labeled with JC-1 as positive control (R1: gated the healthy HUVECs with J-aggregates, R2:gated the unhealthy HUVECs with J-monomers); (B) The percentages of HUVECs with low $\Delta \psi_{m}$ after QDs treatment were detected by flow cytometer; (C) Visualization of mitochondrial structures. Cells are stained with Mitotracker Red FM and the morphological changes of mitochondria were observed by confocal microscopy. Data represent mean $\pm \mathrm{SD}$ of six determinations. ${ }^{*} p<0.05$ and ${ }^{* *} p<0.01$ compared with control. Bars $=50$ $\mu \mathrm{m}$. 

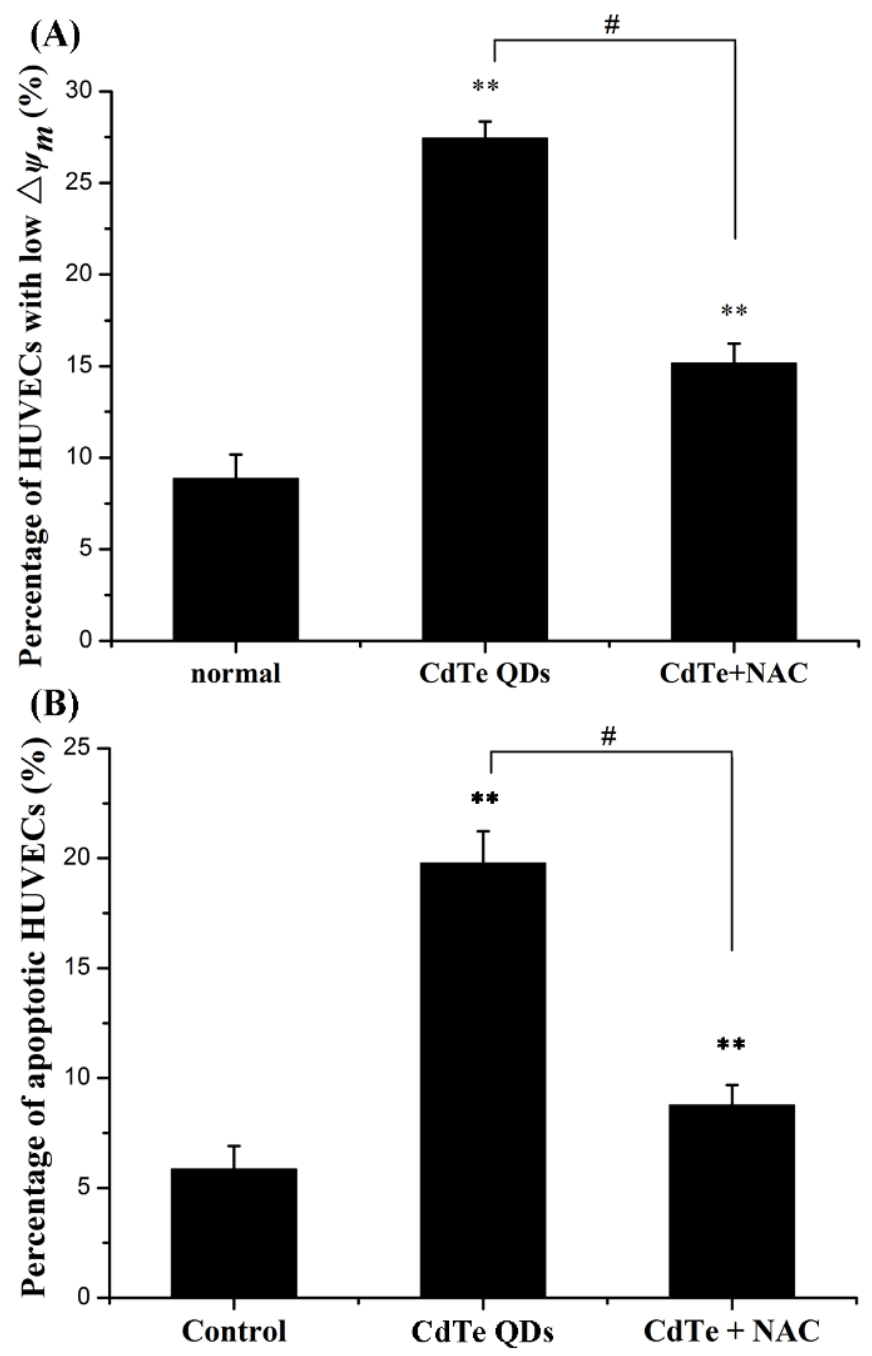

Fig.7 ROS scavenger NAC The inhibited the CdTe QDs-induced (A) mitochondrial depolarization and (B) apoptosis of HUVECs. Data were represented as mean \pm SD of six determinations. ${ }^{* *} p<0.01$ compared with control. 

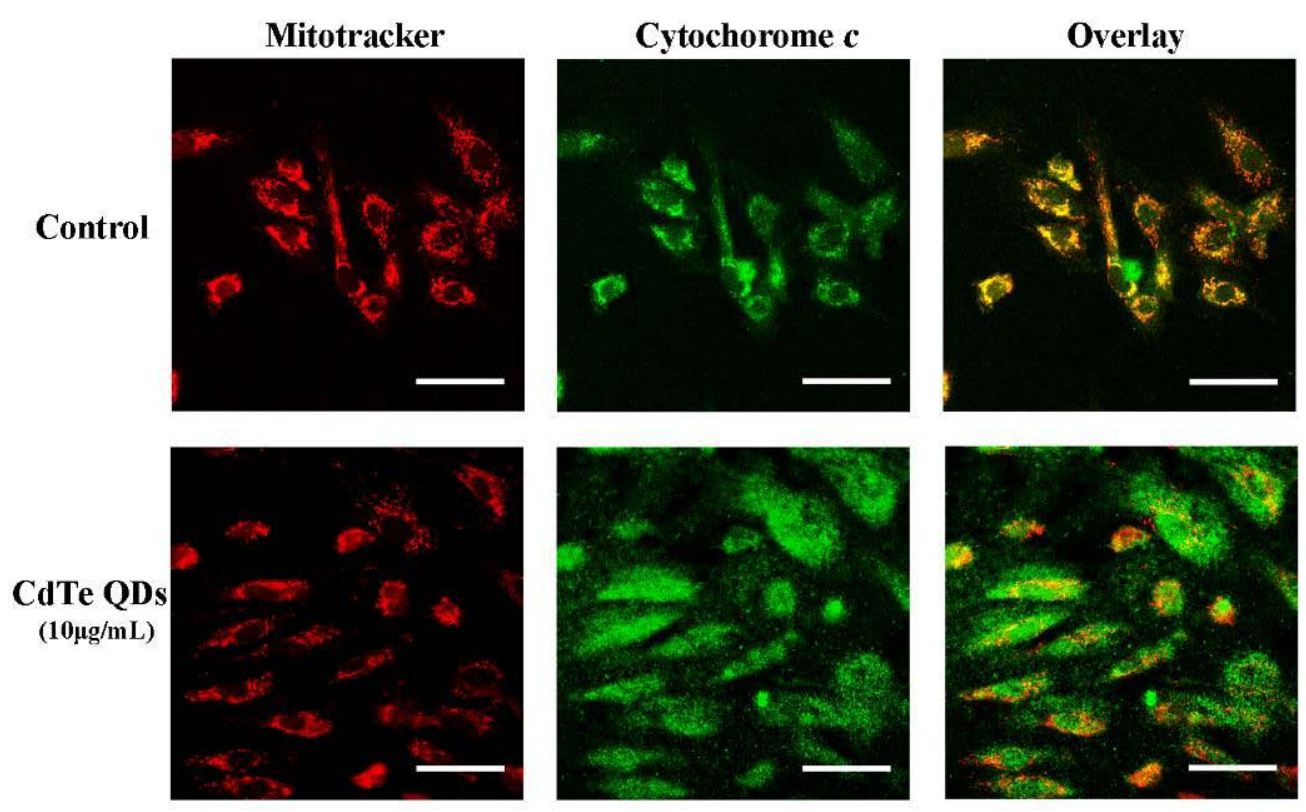

Fig.8 Detection of cytochrome $c$ leakage after 24 h CdTe QDs treatment. Double-labeling immunofluorescence staining of cytochrome $c$ and mitochondria under normal and $10 \mu \mathrm{g} / \mathrm{mL} \mathrm{CdTe}$ QDs treated conditions. Bars $=50 \mu \mathrm{m}$. 
(A)
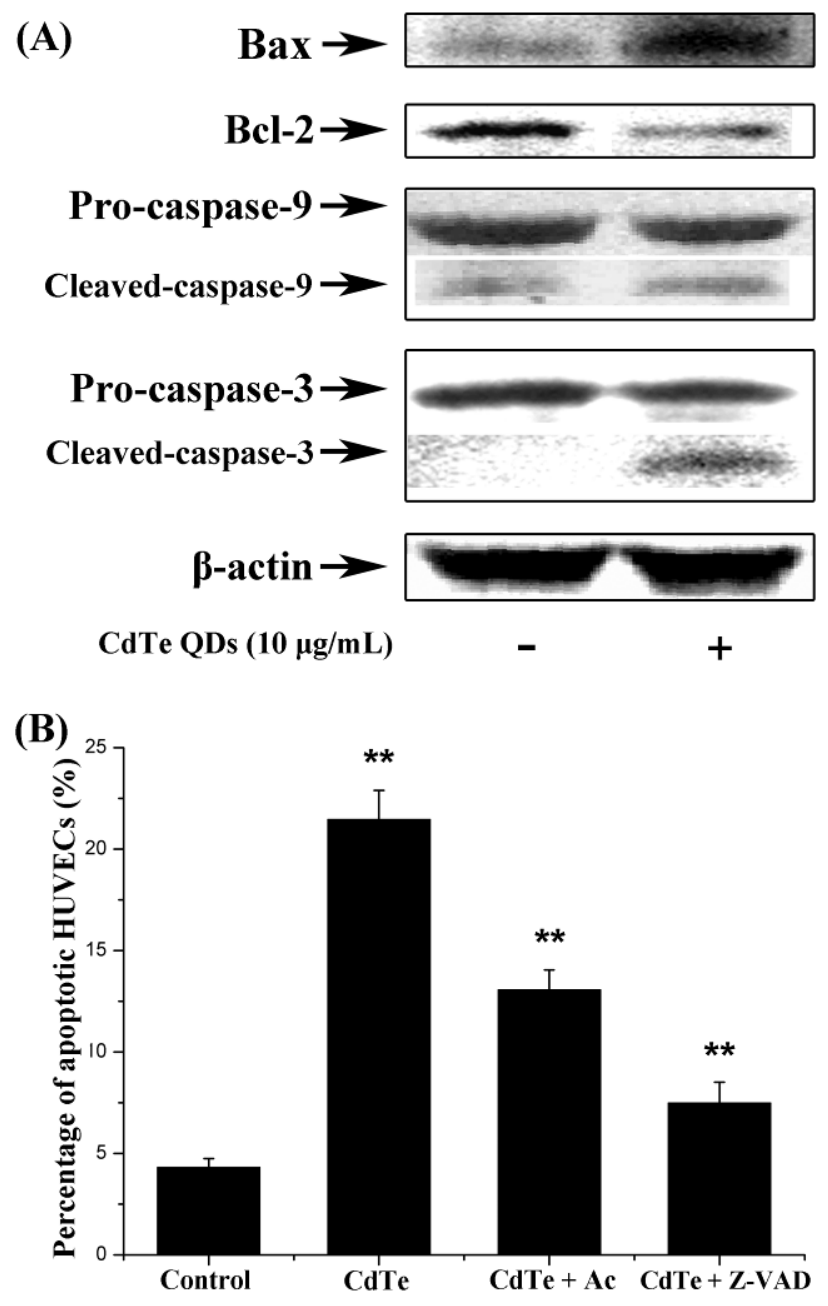

Fig.9 CdTe QDs induce activation of mitochondrial apoptotic pathways. (A) Representative immunoblotting of Bax, Bcl-2, procaspase-9/activated caspase-9 and procaspase-3/activated caspase-3 proteins in HUVECs from both control and $10 \mu \mathrm{g} / \mathrm{mL}$ CdTe QDs-treated groups. (B) The inhibition of Z-VAD-FMK and Ac-DEVE-CHO in CdTe QDs-induced apoptosis of HUVECs. Data were represented as mean \pm SD of six determinations. ${ }^{* *} p<0.01$ compared with control. 


\section{References}

Ekimov, A.I., Efros, A.L., Onushchenko, A.A., 1985. Quantum Size Effect in Semiconductor Microcrystals. Solid State Commun 56, 921-924.

Rossetti, R., Nakahara, S., Brus, L.E., 1983. Quantum Size Effects in the Redox Potentials, Resonance Raman-Spectra, and Electronic-Spectra of Cds Crystallites in Aqueous-Solution. J Chem Phys 79, 1086-1088.

Coe, S., Woo, W.K., Bawendi, M., Bulovic, V., 2002. Electroluminescence from single monolayers of nanocrystals in molecular organic devices. Nature 420, 800-803.

Huffaker, D.L., Park, G., Zou, Z., Shchekin, O.B., Deppe, D.G., 1998. 1.3 mu m room-temperature GaAs-based quantum-dot laser. Appl Phys Lett 73, 2564-2566.

Huynh, W.U., Dittmer, J.J., Alivisatos, A.P., 2002. Hybrid nanorod-polymer solar cells. Science 295, 2425-2427.

Likharev, K.K., 1999. Single-electron devices and their applications. P Ieee 87, 606-632.

Resch-Genger, U., Grabolle, M., Cavaliere-Jaricot, S., Nitschke, R., Nann, T., 2008. Quantum dots versus organic dyes as fluorescent labels. Nature Methods 5, 763-775.

Bagalkot, V., Zhang, L., Levy-Nissenbaum, E., Jon, S., Kantoff, P.W., Langer, R., Farokhzad, O.C., 2007. Quantum dot - Aptamer conjugates for synchronous cancer imaging, therapy, and sensing of drug delivery based on Bi-fluorescence resonance energy transfer. Nano Lett 7, 3065-3070.

Manabe, N., Hoshino, A., Liang, Y.Q., Goto, T., Kato, N., Yamamoto, K., 2006. Quantum dot as a drug tracer in vivo. Ieee T Nanobiosci 5, 263-267.

Tang, M.L., Xing, T.R., Zeng, J., Wang, H.L., Li, C.C., Yin, S.T., Yan, D., Deng, H.M., Liu, J., Wang, M., Chen, J.T., Ruan, D.Y., 2008. Unmodified CdSe quantum dots induce elevation of cytoplasmic calcium levels and impairment of functional properties of sodium channels in rat primary cultured hippocampal neurons. Environmental Health Perspectives 116, 915-922.

Shiohara, A., Hoshino, A., Hanaki, K., Suzuki, K., Yamamoto, K., 2004. On the cyto-toxicity caused by quantum dots. Microbiology and Immunology 48, 669-675.

Chang, S.Q., Dai, Y.D., Kang, B., Han, W., Mao, L., Chen, D., 2009. UV-enhanced cytotoxicity of thiol-capped CdTe quantum dots in human pancreatic carcinoma cells. Toxicology Letters 188, 104-111.

Lovric, J., Bazzi, H.S., Cuie, Y., Fortin, G.R.A., Winnik, F.M., Maysinger, D., 2005. Differences in subcellular distribution and toxicity of green and red emitting CdTe quantum dots. Journal of Molecular Medicine-Jmm 83, 377-385.

Yu, W.W., Qu, L.H., Guo, W.Z., Peng, X.G., 2003. Experimental determination of the extinction coefficient of CdTe, CdSe, and CdS nanocrystals. Chem Mater 15, 2854-2860.

Fu, T., Qin, H.Y., Hu, H.J., Hong, Z., He, S.L., 2010. Aqueous Synthesis and Fluorescence-Imaging Application of CdTe/ZnSe Core/Shell Quantum Dots with High Stability and Low Cytotoxicity. Journal of Nanoscience and Nanotechnology 10, 1741-1746.

Peng, Z.A., Peng, X.G., 2002. Nearly monodisperse and shape-controlled CdSe nanocrystals via alternative routes: Nucleation and growth. J Am Chem Soc 124, 3343-3353.

Ballou, B., Ernst, L.A., Andreko, S., Harper, T., Fitzpatrick, J.A.J., Waggoner, A.S., Bruchez, M.P., 2007. Sentinel lymph node imaging using quantum dots in mouse tumor models. Bioconjugate Chem 18, 389-396.

Ballou, B., Lagerholm, B.C., Ernst, L.A., Bruchez, M.P., Waggoner, A.S., 2004. Noninvasive imaging 
of quantum dots in mice. Bioconjugate Chem 15, 79-86.

Cai, W., Shin, D.W., Chen, K., Gheysens, O., Cao, Q., Wang, S.X., Gambhir, S.S., Chen, X., 2006. Peptide-labeled near-infrared quantum dots for imaging tumor vasculature in living subjects. Nano Lett 6, 669-676.

Gao, X.H., Cui, Y.Y., Levenson, R.M., Chung, L.W.K., Nie, S.M., 2004. In vivo cancer targeting and imaging with semiconductor quantum dots. Nat Biotechnol 22, 969-976.

Jayagopal, A., Russ, P.K., Haselton, F.R., 2007. Surface engineering of quantum dots for in vivo vascular Imaging. Bioconjugate Chem 18, 1424-1433.

Larson, D.R., Zipfel, W.R., Williams, R.M., Clark, S.W., Bruchez, M.P., Wise, F.W., Webb, W.W., 2003. Water-soluble quantum dots for multiphoton fluorescence imaging in vivo. Science 300, 1434-1436.

Tada, H., Higuchi, H., Wanatabe, T.M., Ohuchi, N., 2007. In vivo real-time tracking of single quantum dots conjugated with monoclonal anti-HER2 antibody in tumors of mice. Cancer Res 67, 1138-1144.

Chen, J.Y., Lee, Y.M., Zhao, D., Mak, N.K., Wong, R.N.S., Chan, W.H., Cheung, N.H., 2010. Quantum Dot-mediated Photoproduction of Reactive Oxygen Species for Cancer Cell Annihilation. Photochem Photobiol 86, 431-437.

Choi, A.O., Cho, S.J., Desbarats, J., Lovric, J., Maysinger, D., 2007. Quantum dot-induced cell death involves Fas upregulation and lipid peroxidation in human neuroblastoma cells. J Nanobiotechnology 5, 1.

Su, Y.Y., He, Y., Lu, H.T., Sai, L.M., Li, Q.N., Li, W.X., Wang, L.H., Shen, P.P., Huang, Q., Fan, C.H., 2009. The cytotoxicity of cadmium based, aqueous phase - Synthesized, quantum dots and its modulation by surface coating. Biomaterials 30, 19-25.

Su, Y.Y., Hu, M., Fan, C.H., He, Y., Li, Q.N., Li, W.X., Wang, L.H., Shen, P.P., Huang, Q., 2010. The cytotoxicity of CdTe quantum dots and the relative contributions from released cadmium ions and nanoparticle properties. Biomaterials 31, 4829-4834.

Wu, C.H., Shi, L.X., Li, Q.N., Jiang, H., Selke, M., Ba, L., Wang, X.M., 2010. Probing the Dynamic Effect of Cys-CdTe Quantum Dots toward Cancer Cells in Vitro. Chem Res Toxicol 23, 82-88.

Samia, A.C.S., Chen, X.B., Burda, C., 2003. Semiconductor quantum dots for photodynamic therapy. J Am Chem Soc 125, 15736-15737. 